\title{
Retroperitoneoscopic drainage of a retroperitoneal abscess when open surgery is not an option: a novel surgical technique
}

\begin{abstract}
Objective: An open cholecystectomy has specific surgical indications, including cirrhosis, vesicular cancer, and severe adherence syndrome. Abscesses resulting from the open surgery corresponds to $1 \%$ of complications. When located in the retroperitoneum, these abscesses are life-threatening conditions, having a high mortality from a delayed diagnosis or incomplete drainage. Treatment can be conservative, surgical or endoscopically.
\end{abstract}

Materials and Methods: In the right lateral decubitus position, between the medial and posterior axillary lines, inferior to the twelfth costal space an incision is made for the $12 \mathrm{~mm}$ trocar. Avulsion and resection of the lateral abdominal muscles and latissimus dorsi muscle until inside the retroperitoneum, where under direct vision, the $5 \mathrm{~mm}$ and the pneumatic trocar are inserted above and anterior to the iliac crest.

Results: Through the retroperitoneum scope, the retroperitoneum space is easily accessed to ensure complete abscess drainage. In the out-patient consults, there were no resulting complications reported.

Discussion: Retroperitoneal infections are a surgical and diagnostic challenge. The abscess classification depends on the anatomic location which can direct any of the therapeutic options, between the conservative, surgical, or percutaneous drainage. The conservative approach is reserved for small collections $(<3 \mathrm{~cm})$, the percutaneous is widely used but not exempt from complications, and more recently the minimally invasive approach, the retroperitoneoscopic drainage, has shown superior benefits, with complete drainage and debridement leading to a decreased recurrence rate

Conclusions: We propose a retroperitoneum scope access as a safe and ideal method to drain retroperitoneal abscesses in patients whom a conservative approach cannot be performed, or those who cannot undergo a percutaneous or open surgical technique.

Keywords: open cholecystectomy, retroperitoneal abscess, retroperitoneoscopy, percutaneous drainage, open drainage
Volume 9 Issue 3 - 2019

\author{
Manuel Santiago Mosquera,' Maria Antonia \\ Ocampo, ${ }^{3}$ Alejandro González-Orozco, ${ }^{3}$ \\ Carlos J -Perez Rivera, ${ }^{3}$ Paulo Cabrera ${ }^{2}$ \\ 'Department of Gastrointestinal Surgery, Fundación \\ Cardioinfantil - IC, Colombia \\ ${ }^{2}$ Department of General Surgery, Fundación Cardioinfantil - IC, \\ Colombia \\ ${ }^{3}$ General Surgery Research Group, Fundación Cardioinfantil - \\ IC, Colombia
}

Correspondence: Carlos J-Perez Rivera, General Surgery Research Group, Fundación Cardioinfantil - IC, Bogota, Colombia, Tel + 57 (03I) 6672727 ,

Email cjperezrivera@gmail.com

Received: May 25, 2019 | Published: June 07, 2019

\section{Abbreviations: $\mathrm{CT}$, computed tomography}

\section{Introduction}

Symptomatic cholelithiasis remains a large health problem in the world, typically treated with a laparoscopic cholecystectomy. ${ }^{1}$ Dating back to more than 100 years ago, an open cholecystectomy approach remains a viable alternative to this day, despite the rise of minimally invasive techniques since the 1990s. Most of the open cholecystectomy cases are reduced to hepatic cirrhosis, vesicular cancer and/or severe adherence syndrome. ${ }^{2,3}$ When this approach is preferred, the downside is the increased risk of complications (9$16 \%$ ) and higher morbi-mortality rate of $0.17 \% 0^{2,3}$ The most common complications range from a biliary tract stricture, cholangitis, biliary fistulae or abscess formation secondary to the intestinal flora $(<1 \%){ }^{4}$

Retroperitoneal abscesses are life-threatening conditions, albeit very rare. ${ }^{5}$ The delay in the correct diagnosis and an inefficient drainage can result in septic processes leading to a high morbimortality. ${ }^{6}$ These collections are usually secondary complications of abdominal infections, damage or malignant lesions of retroperitoneal organs, and lastly damage adjacent to osteomyelitis, lymphadenitis, or bacteremia. ${ }^{7}$ The treatment remains the same, between a conservative, a surgical or an endoscopic approach. ${ }^{5}$

Our objective is to present a retroperitoneoscopic approach to drainage of a giant retroperitoneal abscess secondary to an open cholecystectomy in a patient with a difficult surgical abdomen due to multiple previous interventions.

\section{Case presentation}

A 49-year-old male patient, with previous history of splenectomy, chronic renal failure, and left nephrectomy, is admitted due to abdominal pain, predominantly in epigastrium, of 8days associated with multiple emetic episodes and febrile syndrome. On arrival, a computed tomography (CT) scan was performed reporting acute cholecystitis requiring an open cholecystectomy based on the previous interventions presenting a surgical challenge. During the post-operative period, patient had a poor clinical progression leading to a second surgical intervention where a hemoperitoneum of approximately $1500 \mathrm{cc}$ was discovered, without proper visualization 
of the source of bleeding. This led to packing of the abdomen and closure of the incision. Posterior to this, multiple surgical peritoneal lavages were performed until complete closure of the abdomen wall was achieved and the bleeding resolved. However, the clinical course continued to be torpid, whereby a second abdominal CT scan was used, discovering a gastro-hepatic ligament abscess of $15 \times 6 \times 9 \mathrm{~cm}$ in size with an approximate volume of $450 \mathrm{cc}$ (Figure 1) (Figure 2). Given the multiple previous surgical interventions and high risk of a "hostile" abdomen, a retroperitoneoscopic drainage as the ideal treatment.
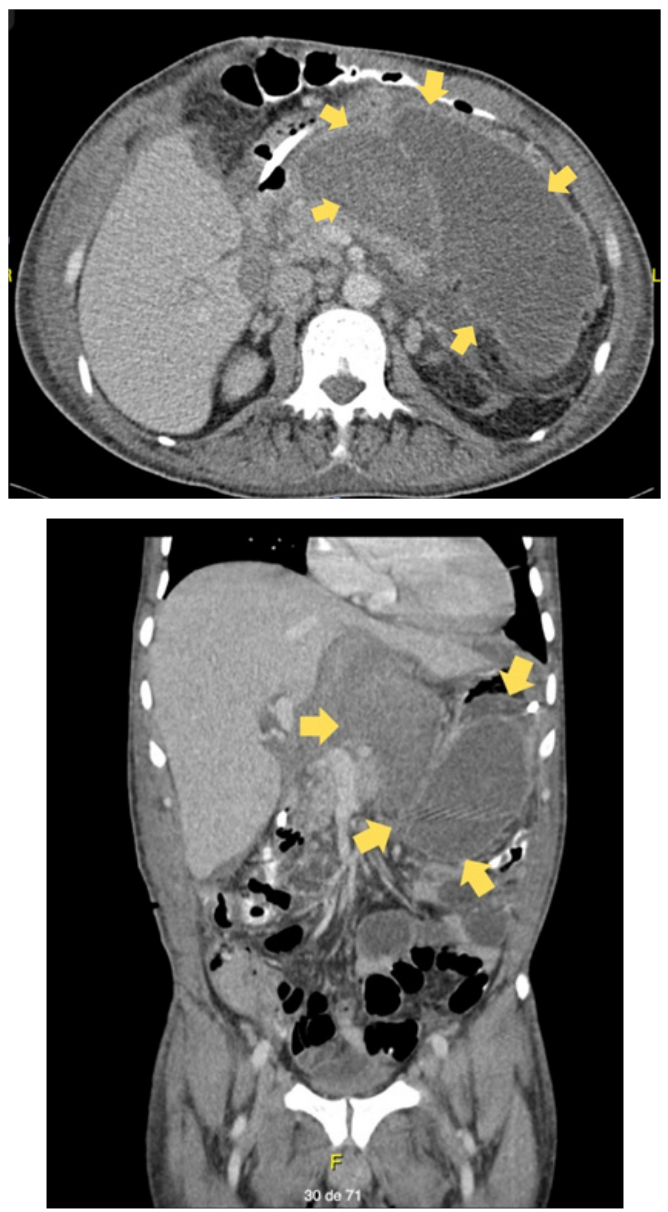

Figure I\&2 Abdominal computed tomography (CT) scan. The arrow represents the fluid collection of $15 \times 6 \times 9 \mathrm{~cm}$ with an approximate volume of 450 cc.

\section{Surgical technique}

In the right lateral decubitus position, between the medial and posterior axillary lines, inferiorly to the twelfth costal space, a $12 \mathrm{~mm}$ trocar is inserted until entrance to the peritoneum (Figure 3). Posteriorly, via direct vision, above the anterior iliac crest, a $5 \mathrm{~mm}$ trocar is placed as well as the pneumoperitoneum trocar until $15 \mathrm{~mm}$ inside the cavity. Then, a retroperitoneal dissection is performed, locating the bulging zone surrounded by a fibrous and thick capsule this was punctured obtaining serous-hematic fluid, blood clots and fibrin. Additionally, the evidence of multiple septaes leads to further insertion of the $12 \mathrm{~mm}$ trocar anteriorly and downwards widening the cavity ensuring a complete debridement. Finally, the cavity is washed with $1000 \mathrm{cc}$ of normal saline solution until clearing of the fluid leading to a closure of the incisional wounds.

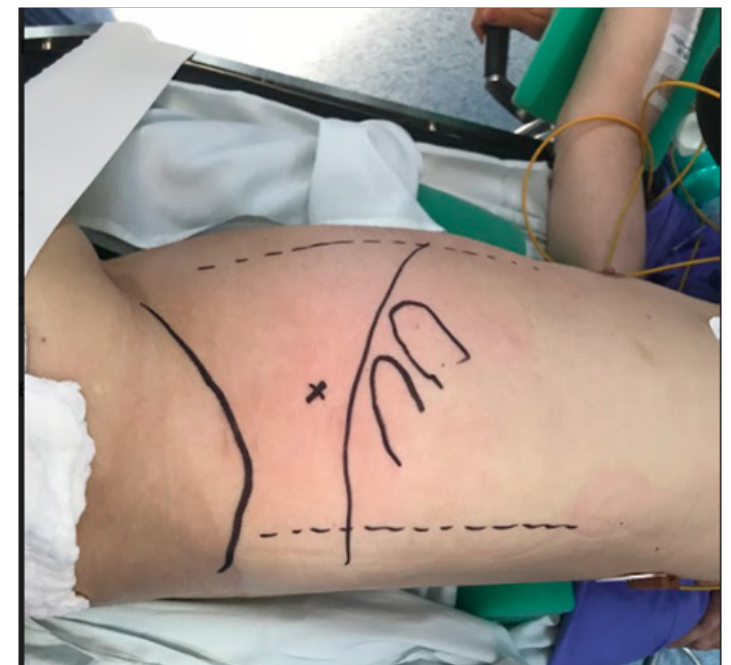

Figure 3 Patient in the right lateral decubitus position. The point marked is the inferior border of the eleventh and twelfth rib, where the incision was performed.

\section{Results}

Given the surgical approach performed, the retroperitoneal access was achieved with complete drainage of the collection. During the post-operative follow-up period, the patient remained stable during the remaining hospital in-stay.

\section{Discussion}

Retroperitoneal infections are a surgical challenge given the diagnostic delay resulting in a high mortality rate, ranging from 22$46 \%$. The retroperitoneal zone is usually divided into three specific spaces: the anterior para-renal space (between the anterior para-renal fascia and the posterior peritoneum), the peri-renal space (delimited by the Gerotas fascia), and the posterior para-renal space (behind the Gerotas fascia). ${ }^{4}$ Retroperitoneal abscesses can be classified according to their anatomic location: peri-nephric, superior, pelvic, or musculoskeletal. ${ }^{7}$ In this case report, the abscess was in the superior retroperitoneal space.

Abscess treatment can be conservative, surgical, or percutaneous. ${ }^{5}$ The conservative approach is indicated in small collections (less than $3 \mathrm{~cm}$ ) and consists of a long-term antibiotic regimen. The percutaneous drainage has become the gold-standard in recent years given its high resolution rate. This option is also ideal due to a lack of invasive surgical risks. However, a percutaneous drainage is not always possible and in these cases an open drainage must be performed.

Within the surgical drainages, the minimally invasive retroperitoneal approach was first described in 1973 by Dr. Wittmoser in a sympathectomy, now known as the retroperitoneoscopic approach. ${ }^{8}$ This technique has shown superior results compared to the percutaneous, open, or trans-abdominal laparoscopic approaches, due to a complete drainage and debridement of the affected tissues, specially the thick wall that often increases the collection recurrence rate. ${ }^{9}$ 
According to J. Creeps et al., ${ }^{6}$ the retroperitoneal approach is much more effective than the trans-peritoneal approach, given that the latter has a higher failure rate of $67 \%$ compared to $15 \%$, making the former the ideal approach for peri-renal and superior collections. Additionally, a complete drainage relies on the adjacent tissues being free of collection-induced infection, which can be controlled more successfully retroperitoneally. ${ }^{6}$

The retroperitoneoscopic approach in superior abscesses is a safe and effective technique given a decreased dissemination of the infection to the peritoneal space as well as ample visualization of both ports, where one is visually assisted (echography) and the other is via direct vision. ${ }^{10}$ Additionally, given its minimally invasive approach, it results as a safer alternative to the percutaneous or open techniques due to a lower in-patient stay as well as a complete drainage reducing the recurrence rate ${ }^{11}$.

\section{Conclusions}

We propose the retroperitoneoscopic drainage as a feasible approach in retroperitoneal abscesses of large sizes in patients whom are not candidates for a conservative, percutaneous, or open surgery approach.

\section{Informed consent statement}

Written consent was obtained from the patient for publication of this report. Any details identifying the individuals to the clinical history and images associated were eliminated as to remain anonymous.

\section{Acknowledgments}

Supported by the General Surgery Research Group at Fundación Cardioinfantil - IC.

\section{Conflicts of interest}

The authors declare they have no conflicts of interest.

\section{References}

1. El Nakeeb A, Mahdy Y, Salem A, et al. Open Cholecystectomy Has a Place in the Laparoscopic Era: a Retrospective Cohort Study. Indian Journal of Surgery. 2017;79(5):437-443.

2. Roslyn J, Binns G, Hughes E, et al. Open Cholecystectomy A Contemporary Analysis of 42,474 Patients. Ann Surg. 1993;218(2):129-137.

3. Jones MW, Deppen JG, Gallbladder. Cholecystectomy. In: Stat Pearls. Treasure Island (FL): Stat Pearls Publishing; 2018.

4. Patel S,HashmiZ, Marx R.Aretroperitoneal abscess caused by Haemophilus parainfluenza after endoscopic retrograde cholangiopancreatography and open cholecystectomy with a common bile duct exploration: a case report. J Med Case Rep. 2010;4(1).

5. Manjon C, Tejido Sanchez A, Piedra Lara J, et al. Retroperitoneal Abscesses Analysis of a Series of 66 Cases. Scand J Urol Nephrol. 2003;37(2):139-144.

6. Crepps J, Welch J, Orlando R. Management and Outcome of Retroperitoneal Abscess. Ann Surg. 1986;205(3): 276-281.

7. Altemeier W, Alexander J. Retroperitoneal Abscess. 18th Annual Meeting of the Central Surgical Association, St Louis, Feb 17, 1961;83(4):512524

8. Rassweiler J, Seemann O, Frede T, et al. Retroperitoneoscopy: Experience With 200 Cases. J Urol. 1998;160(4):1265-1269.

9. Zhang X, Zhang Z, Zhang Y, et al. Minimally invasive retroperitoneoscopic surgery for psoas abscess with thoracolumbar tuberculosis. Surgical Endoscopy. 2015;29(8):2451-2455.

10. Del Moral J, Villegas-Herrera M, Medina-Benítez A, et al. Retroperitoneoscopy to Extract Dropped Gallstones After Laparoscopic Cholecystectomy. J Laparoendosc Adv Surg Tech A. 2006;16(3):290-293.

11. Katara A, Shah R, Bhandarkar D, et al. Retroperitoneoscopic drainage of a psoas abscess. Journal of Pediatric Surgery. 2004;39(9):e4-e5. 